EPJ Web of Conferences 59, 01004 (2013)

DOI: $10.1051 /$ epjconf/20135901004

(C) Owned by the authors, published by EDP Sciences, 2013

\title{
Progress in direct-drive inertial confinement fusion
}

\author{
R.L. McCrory ${ }^{1,2, a}$, D.D. Meyerhofer ${ }^{1,2}$, R. Betti ${ }^{1,2}$, T.R. Boehly ${ }^{1}$, T.J.B. Collins ${ }^{1}$, \\ R.S. Craxton ${ }^{1}$, J.A. Delettrez ${ }^{1}$, D.H. Edgell ${ }^{1}$, R. Epstein ${ }^{1}$, D.H. Froula ${ }^{1}$, \\ V.Yu. Glebov ${ }^{1}$, V.N. Goncharov ${ }^{1}$, D.R. Harding ${ }^{1}$, S.X. Hu ${ }^{1}$, I.V. Igumenshchev ${ }^{1}$, \\ J.P. Knauer ${ }^{1}$, S.J. Loucks ${ }^{1}$, J.A. Marozas ${ }^{1}$, F.J. Marshall ${ }^{1}$, P.W. McKenty ${ }^{1}$, \\ T. Michel ${ }^{1}$, P.M. Nilson ${ }^{1}$, P.B. Radha ${ }^{1}$, S.P. Regan ${ }^{1}$, T.C. Sangster ${ }^{1}$, W. Seka ${ }^{1}$, \\ W.T. Shmayda ${ }^{1}$, R.W. Short ${ }^{1}$, D. Shvarts ${ }^{1,3}$, S. Skupsky ${ }^{1}$, J.M. Soures ${ }^{1}$, \\ C. Stoeckl ${ }^{1}$, W. Theobald ${ }^{1}$, B. Yaakobi ${ }^{1}$, J.A. Frenje ${ }^{4}$, D.T. Casey ${ }^{4}$, C.K. Li ${ }^{4}$, \\ R.D. Petrasso ${ }^{4}$, F.H. Séguin ${ }^{4}$, S.J. Padalino ${ }^{5}$, K.A. Fletcher ${ }^{5}$, P.M. Celliers ${ }^{6}$, \\ G.W. Collins ${ }^{6}$ and H.F. Robey ${ }^{6}$
}

${ }^{1}$ Laboratory for Laser Energetics, 250 East River Road, Rochester, NY 14623-1299, USA

${ }^{2}$ Departments of Mechanical Engineering and Physics, University of Rochester, Rochester, NY, USA

${ }^{3}$ Nuclear Research Center, Negev, Israel

${ }^{4}$ Plasma Science and Fusion Center, Massachusetts Institute of Technology, Cambridge, MA, USA

${ }^{5}$ State University of New York - Geneseo, Geneseo, NY, USA

${ }^{6}$ Lawrence Livermore National Laboratory, Livermore, CA, USA

\begin{abstract}
Significant progress has been made in direct-drive inertial confinement fusion research at the Laboratory for Laser Energetics since the 2009 IFSA Conference [R.L. McCrory et al., J. Phys.: Conf. Ser. 244, 012004 (2010)]. Areal densities of $300 \mathrm{mg} / \mathrm{cm}^{2}$ have been measured in cryogenic target implosions with neutron yields $15 \%$ of 1-D predictions. A model of crossed-beam energy transfer has been developed to explain the observed scattered-light spectrum and laser-target coupling. Experiments show that its impact can be mitigated by changing the ratio of the laser beam to target diameter. Progress continues in the development of the polar-drive concept that will allow direct-drive-ignition experiments to be conducted on the National Ignition Facility using the indirect-drive-beam layout.
\end{abstract}

\section{INTRODUCTION}

The direct-drive approach to inertial confinement fusion (ICF) [1] provides an attractive alternative to the indirect-drive approach [2] that is currently being pursued on the National Ignition Facility (NIF) [3]. Direct-drive ICF research is carried out by the Laboratory for Laser Energetics (LLE) using the OMEGA Laser System [4]. LLE's research includes symmetric implosions of cryogenic [5] and warm surrogate [6] targets as well as the development of polar drive [7]. Polar drive allows directdrive-ignition experiments [7] to be performed on the NIF [3] using the indirect-drive beam layout, significantly reducing the cost of performing these experiments.

ae-mail: rmcc@lle.rochester.edu

This is an Open Access article distributed under the terms of the Creative Commons Attribution License 2.0, which permits unrestricted use, distribution, and reproduction in any medium, provided the original work is properly cited. 


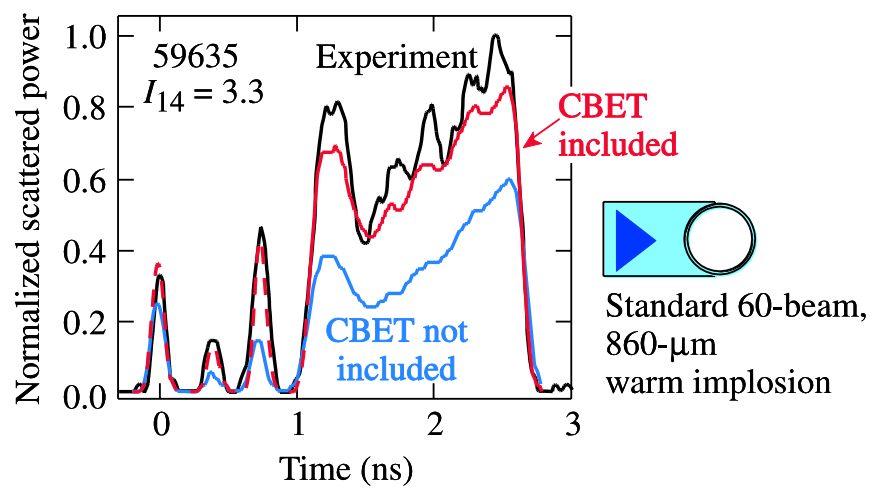

Figure 1. Comparison of the measured and predicted power histories of the light scattered at the incident laser wavelength for the experiments (black), without CBET (blue) and with CBET (red).

Crossed-beam energy transfer (CBET) [8-10] has been shown to be important in direct-drive experiments [11]. Simulations and experiments have shown that reducing the laser beam's diameter relative to the target diameter improves the laser-target coupling, increasing the implosion velocity.

This document describes recent direct-drive progress, starting with understanding CBET (Section 2), cryogenic target implosions (Section 3), and the development of the polar-drive-ignition concept (Section 4). A summary is provided in Section 5.

\section{CROSSED-BEAM ENERGY TRANSFER}

Crossed-beam energy transfer (CBET) [8-10] has been identified as a source of reduced laser absorption in direct-drive experiments [11]. Laser light that is transmitted around the target can interact with the central region of incident beams to scatter light from the Mach-1 surface, reducing the ontarget absorbed energy. The Mach-1 surface occurs near quarter-critical electron density $\left(n_{\mathrm{e}}=2\right.$ to $3 \times 10^{21} \mathrm{~cm}^{3}$ ), where the electron temperature $T_{\mathrm{e}} \sim 2 \mathrm{keV}$. The spectrum of the scattered light is consistent with this analysis [12]. Figure 1 compares the time-resolved measured scattered light from spherical target implosions with those predicted by 1-D simulations that include and do not include CBET. When CBET is included, the simulations are in much better agreement with the experimental measurements. CBET is modeled in 1-D simulations (including 3-D ray tracing and known focal-spot profiles) by considering pair-wise interactions of rays and calculating the energy transfer between rays using the stimulated Brillouin scattering gain rate for each pair [11]. The standard OMEGA phase plates [13] with a nominal diameter of $860 \mu \mathrm{m}$ were used in these experiments. With this phase-plate design, $\sim 5 \%$ of the incident laser light propagates around the target.

\subsection{Modifications of CBET by changing the laser beam relative to the target diameter}

Figure 2 shows the predicted scattered-light energy as a function of the ratio of the laser beam to target diameter. As this ratio is decreased, the scattered light is predicted to decrease as well, although the expected on-target nonuniformities increase (black curve and right hand axis). The decrease in scattered light is caused directly by reducing the energy missing the target (orange dashed) and by reducing CBET because less beam energy that misses the target reaches the Mach-1 surface (blue solid).

A series of experiments were performed using a set of phase plates with smaller focal spots to drive the implosion of $860-\mu$ m-diam, $20-\mu$ m-thick $\mathrm{CH}$ shells filled with 10 atm of $\mathrm{D}_{2}$. The beam radius was varied by defocusing the phase plates. Figure 3 shows the changes in the absorption and neutron bang time as a function of the ratio of the beam to target radius. As expected from Figure 2, the 


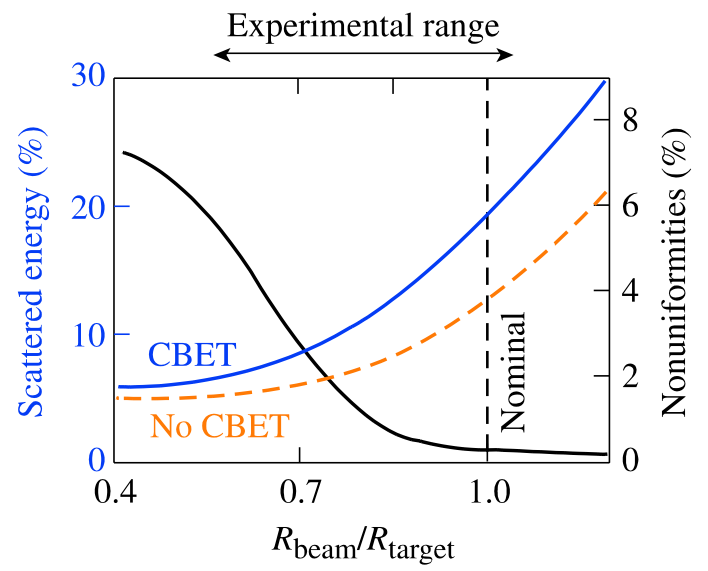

Figure 2. Predicted scattered-light energy fraction as a function of the ratio of the laser beam radius to the target radius with (blue) and without (orange) CBET included. The right axis shows the predicted on-target beam nonuniformity (black).
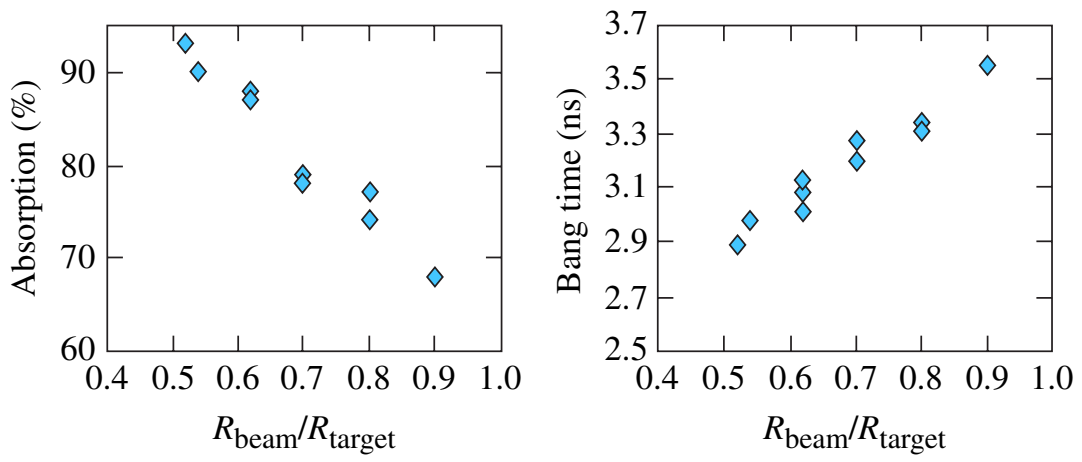

Figure 3. Absorption (left) and bang time (right) as a function of the ratio of the beam to target radius for a series of OMEGA plastic-shell implosions.

absorption increases with decreasing beam radius, while the neutron bang time comes earlier, indicating a $>25 \%$ increase in implosion velocity. Changes in the scattered-light spectrum confirmed the reduction in CBET. The single-beam nonuniformity of the smaller phase plates is not optimal for studying the effects of increased target velocity on the neutron yield. The results suggest that cryogenic target performance can be improved by increasing the target diameter by $10 \%$ to $20 \%$ using the standard OMEGA phase plates that provide a much more uniform irradiation pattern than the phase plates used for the experiments shown in Figure 3.

\section{OMEGA CRYOGENIC IMPLOSIONS}

Direct-drive implosions of cryogenic-deuterium-tritium (DT) targets that are hydrodynamically equivalent to NIF ignition targets are an essential part of developing NIF polar-drive ignition [1, 5, 14]. Recent implosions have shown areal densities up to $300 \mathrm{mg} / \mathrm{cm}^{2}[5,14]$, which are close to those predicted by 1-D simulations that include the effects of CBET. Figure 4 compares the measured areal density in cryogenic target implosions with the predictions of 1-D simulations that include the CBET 


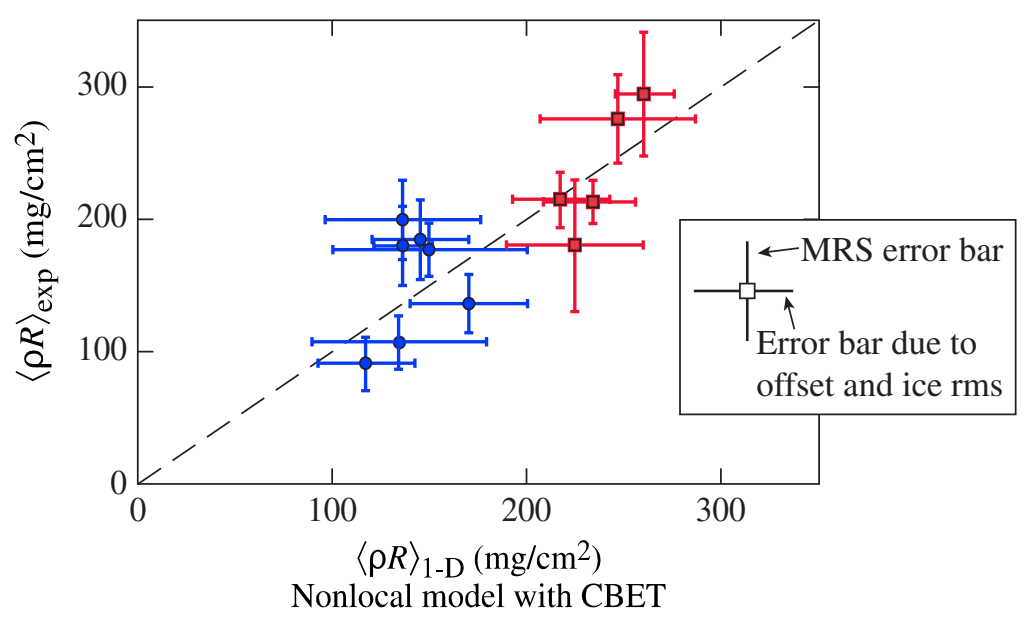

Figure 4. Comparison of measured and simulated areal densities for cryogenic implosions with predicted adiabats of 2.5 (blue) and 2.0 (red).

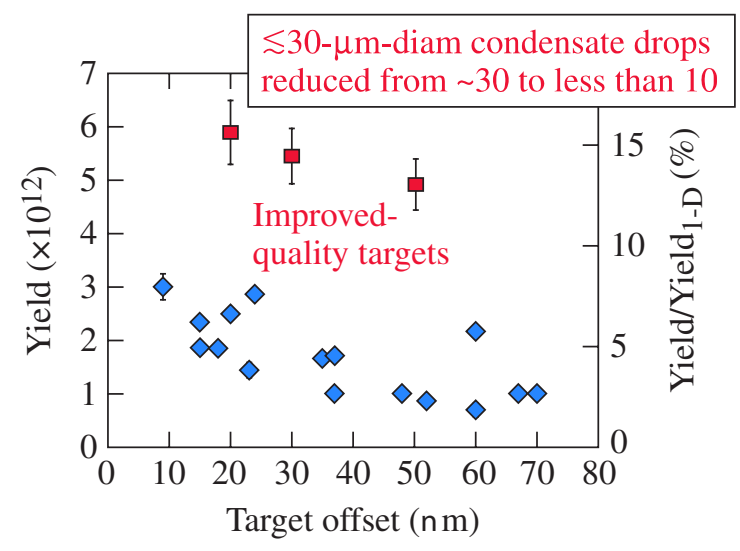

Figure 5. The measured neutron yield and yield relative to 1-D simulations in cryogenic target implosions as a function of target offset from target chamber center, before (blue) and after (red) reducing the surface contamination.

effects for two triple-picket pulse shapes that are predicted to have slightly different adiabats [5]. The adiabat is defined as the ratio of the shell pressure to the Fermi-degenerate pressure at the same density [15]. The blue and red points have predicted adiabats of 2.5 and 2.0, respectively. Good agreement is observed up to the highest areal densities [5, 14].

Significant effort has been devoted to improving the surface quality of cryogenic targets. One major source of surface contamination is $\sim 30-\mu$ m-diam droplets caused by hydrocarbons freed from the surfaces of the piping during the high-pressure DT fill. By pre-cleaning the lines (taking them to high pressure and then pumping before the fill), the number of observed defects on the target surface was significantly reduced, from $\sim 30$ per target to fewer than 10 . Figure 5 shows the neutron yield measured in cryogenic target implosions as a function of target offset [16] from target chamber center, before (blue) and after (red) reducing the surface contamination. The yield along with the yield relative to 1-D predictions increases by a factor of 2 to 3 . The highest-performing targets produce yields that are $\sim 15 \%$ of 1-D predictions. 

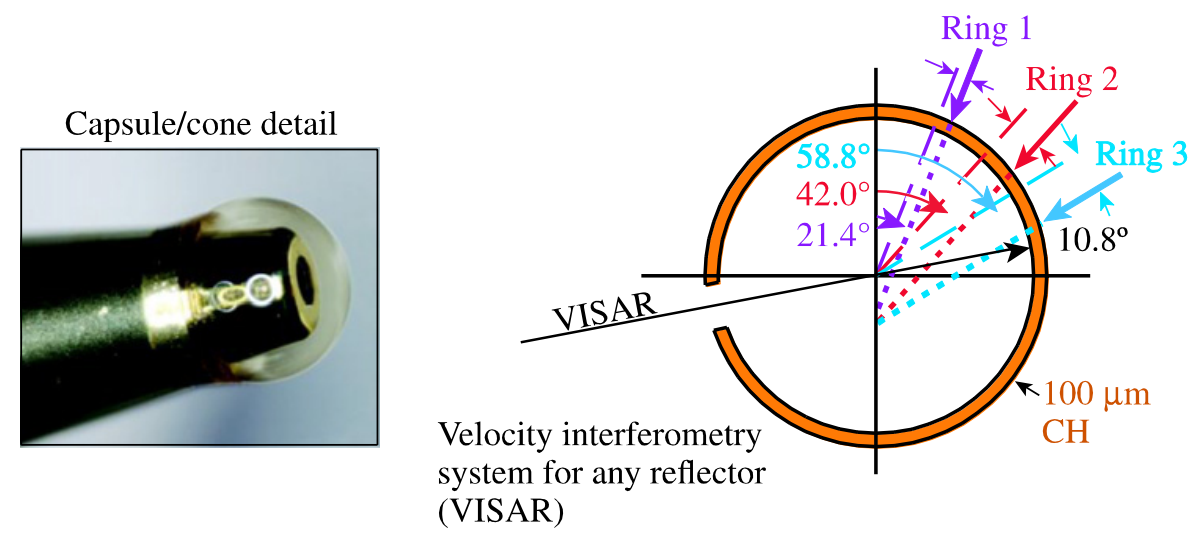

Figure 6. Cone-in-shell shock-timing target (left) and the OMEGA polar-drive beam orientation (right) with velocity interferometry system for any reflector (VISAR) viewing axis shown.

\section{POLAR DRIVE}

Polar-drive ignition allows direct-drive-ignition experiments [7] to be performed on the NIF [3] using the indirect-drive beam layout. This will significantly reduce the cost of performing these experiments. Initial polar-drive target designs show a gain of 32 in 2-D simulations when all expected nonuniformity sources are included [17]. Experiments are being performed on OMEGA to develop and validate the polar-drive concept [18-20]. Polar-drive experiments involve repointing 40 OMEGA beams to a configuration that is similar to that envisioned for NIF experiments.

\subsection{Shock timing}

A major design issue for polar drive is understanding the coupling of the high angle of incidence beams that irradiate the equatorial region of the target. The cone-in-shell shock-timing technique, successfully used to time shock waves on OMEGA and NIF experiments [21], is being used to measure the equatorial shock waves produced by a multiple-picket drive pulse on OMEGA. Figure 6 shows an example of the cone-in-shell target used for shock-timing experiments (left) and the polar-drive-irradiation geometry (right).

Figure 7 shows the triple-picket pulse shape used to drive the equatorial shock waves (left) and a comparison of the measured and predicted shock velocities in a 100- $\mu$ m-thick $\mathrm{CH}$ (warm) target (right). The shock wave is not observed for the first $\sim 3$ ns because of the blanking of the VISAR window [21]. After the window becomes transparent, the measured shock velocities are in good agreement with the simulations, suggesting that the equatorial coupling is well modeled.

\subsection{Compression results}

A variant of the triple-picket pulse shape used for cryogenic target implosions (Section 3) was used in warm target polar-drive implosions on OMEGA. Areal densities up to $125 \mathrm{mg} / \mathrm{cm}^{2}$ were measured, in good agreement with 1-D hydrodynamic simulations [20]. The areal densities are lower than those in the cryogenic implosions described in Section 3 because more massive targets were driven by only 40 of the 60 OMEGA beams. 

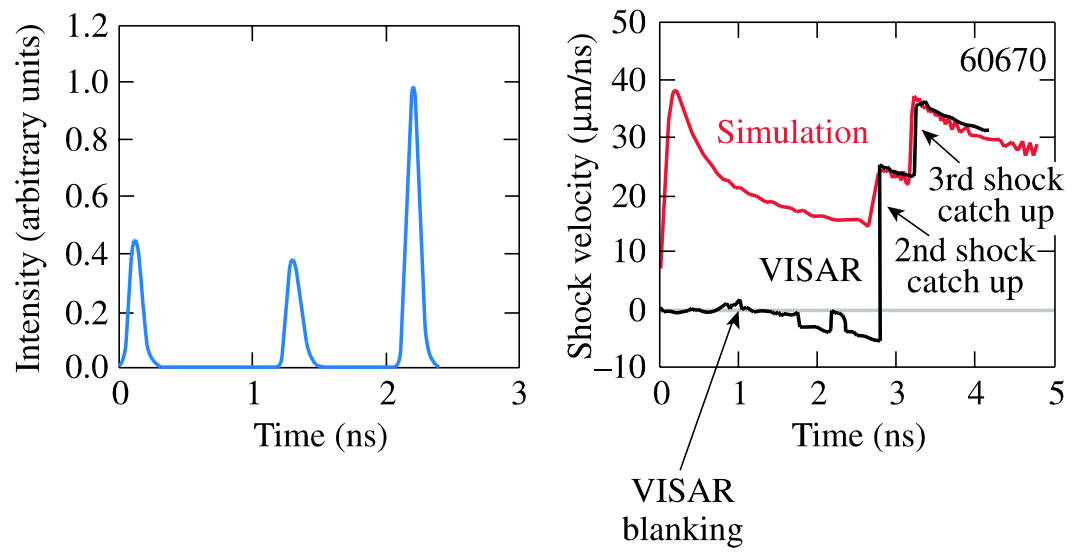

Figure 7. Pulse shape used to measure the equatorial shock timing in the polar-drive configuration shown in Figure 6 (left). The right panel shows good agreement between the measured and simulated shock velocities in a $100 \mu$ m-thick $\mathrm{CH}$ spherical target.

\section{SUMMARY}

Significant progress has been made in the direct-drive inertial confinement fusion concept since the 2009 IFSA meeting [22]. The effects of crossed-beam energy transfer have been quantified and mitigation strategies have been identified. Cryogenic target implosions have shown improved areal densities and neutron yields. Further research on the polar-drive-ignition concept shows promising results. These results give confidence that polar-drive ignition is achievable on the National Ignition Facility within the next decade.

This work was supported by the U.S. Department of Energy Office of Inertial Confinement Fusion under Cooperative Agreement No. DE-FC52-08NA28302, the University of Rochester, and the New York State Energy Research and Development Authority. The support of DOE does not constitute an endorsement by DOE of the views expressed in this article.

\section{References}

[1] R.L. McCrory et al., Phys. Plasmas 15, 055503 (2008)

[2] S.W. Haan et al., Phys. Plasmas 18, 051001 (2011)

[3] E.I. Moses, Fusion Sci. Technol. 54, 361-366 (2008)

[4] T.R. Boehly et al., Opt. Commun. 133, 495-506 (1997)

[5] V.N. Goncharov et al., Phys. Rev. Lett. 104, 165001 (2010)

[6] P.B. Radha et al., Phys. Plasmas 12, 032702 (2005)

[7] S. Skupsky et al., Phys. Plasmas 11, 2763-2770 (2004)

[8] P. Michel et al., Phys. Plasmas 16, 042702 (2009)

[9] P. Michel et al., Phys. Plasmas 17, 056305 (2010)

[10] P. Michel et al., Phys. Rev. Lett. 102, 025004 (2009)

[11] I.V. Igumenshchev et al., Phys. Plasmas 17, 122708 (2010)

[12] D.H. Edgell et al., Bull. Am. Phys. Soc. 54, 145 (2009)

[13] Y. Lin et al., Opt. Lett. 20, 764-766 (1995) 


\section{IFSA 2011}

[14] T.C. Sangster et al., Phys. Plasmas 17, 056312 (2010)

[15] J.D. Lindl, Inertial confinement fusion: The quest for ignition and energy gain using indirect drive (Springer-Verlag, New York, 1998), Chap. 6, pp. 61-82

[16] S.X. Hu et al., Phys. Plasmas 17, 102706 (2010)

[17] T.J.B. Collins et al., "Preparing for polar drive at the National Ignition Facility," presented at the 53rd Annual Meeting of the APS Division of Plasma Physics, Salt Lake City, UT, 14-18 November 2011

[18] F.J. Marshall et al., J. Phys. IV France 133, 153-157 (2006)

[19] R.S. Craxton et al., Phys. Plasmas 12, 056304 (2005)

[20] P. B. Radha et al., these proceedings

[21] T.R. Boehly et al., Phys. Plasmas 16, 056302 (2009)

[22] R.L. McCrory et al., J. Phys.: Conf. Ser. 244, 012004 (2010) 